EUROPEAN ORGANIZATION FOR NUCLEAR RESEARCH

CERN - PS DIVISION

CERN/PS 99-58 (HP)

\title{
Effect of a Biased Probe on the Afterglow Operation of an ECR4 Ion Source
}

\author{
C.E. Hill, D. Küchler, F. Wenander, B.H. Wolf
}

\begin{abstract}
Various experiments have been performed on a $14.5 \mathrm{GHz}$ ECR4 in order to improve the ion yield. The source runs in pulsed afterglow mode, and provides currents $\sim 120 \mathrm{e} \mu \mathrm{A}$ of $\mathrm{Pb}^{27+}$ to the CERN Heavy Ion Facility on an operational basis. In the search for higher beam intensities, the effects of a pulsed biased disk on axis at the injection side were investigated with different pulse timing and voltage settings.

No proof for absolute higher intensities was seen for any of these modifications. However, the yield from a poorly tuned/low-performing source could be improved and the extracted pulse was less noisy with bias voltage applied. The fast response on the bias implies that increases/decreases are not due to ionisation processes. A good tune for high yield of high charge states during the afterglow coincides with a high plasma potential.
\end{abstract}

Paper presented at the 8th International Conference on Ion sources, ICIS'99, Kyoto, Japan, September 6-10, 1999

Geneva,

29 October 1999 


\section{Effect of a Biased Probe on the Afterglow Operation of an ECR4 Ion Source \\ C. E. Hill, D. Küchler, F. Wenander, B.H. Wolf \\ PS Division, CERN, 1211 Geneva 23 \\ Switzerland}

\begin{abstract}
Various experiments have been performed on a $14.5 \mathrm{GHz}$ ECR4 in order to improve the ion yield. The source runs in pulsed afterglow mode, and provides currents $\sim 120 \mathrm{e} \mu \mathrm{A}$ of $\mathrm{Pb}^{27+}$ to the CERN Heavy Ion Facility on an operational basis. In the search for higher beam intensities, the effects of a pulsed biased disk on axis at the injection side were investigated with different pulse timing and voltage settings.

No proof for absolute higher intensities was seen for any of these modifications. However, the yield from a poorly tuned/low-performing source could be improved and the extracted pulse was less noisy with bias voltage applied. The fast response on the bias implies that increases/decreases are not due to ionisation processes. A good tune for high yield of high charge states during the afterglow coincides with a high plasma potential.
\end{abstract}

\section{Introduction}

The CERN Heavy Ion Accelerating Facility [1], after five periods of operation, has become a reliable injector for the lead ions used by the heavy ion physics experiments. An Electron Cyclotron Resonance Ion Source (ECR-4) operates in the afterglow mode [2] to produce short pulses (500-1500 $\mu \mathrm{s})$ of highly charged ions $\left(\mathrm{Pb}^{27+}\right)$ that are accelerated in the heavy ion linac (Linac3). The maximum intensity obtained from the source, in an exceptionally stable afterglow mode of operation, was more than $120 \mathrm{e} \mu \mathrm{A}$ of $\mathrm{Pb}^{27+}$, extracted at an energy of $2.5 \mathrm{keV} / \mathrm{u}$. The search for higher beam intensities continues as the LHC project [3] requires a pulse current at least an order of magnitude higher. Two different approaches are being tried: either to increase the total number of ions in the plasma, or to extract the available ions in a shorter pulse.

\section{Tests and results with biased axial electrode}

It is often claimed that a negatively biased electrode, positioned on axis in the plasma at the injection side, may reduce the electron losses out of the magnetic bottle and/or influence the electron density positively due to the injection of cold electrons created by bombardment of the electrode with loss electrons and ions. Recent investigations, on the other hand, suggest that the ion yield increase be mainly due to improved extraction conditions [4] or plasma potential optimisation [5].

It has previously been shown in an ECR for sulphur ions (a MiniMafios with off axis microwave coupling) that the presence of a biased electrode in the vicinity of the plasma improved the performance and stabilised the afterglow [6]. On the ECR4, (microwave coupling on axis) a bias could be applied to the inner conductor of the coaxial transition which contains the sample oven (Fig. 1), but no gain in current could be observed [7] at the time.
Figure 2 shows the injection beam line at Linac 3. The ion intensity was measured in Faraday cups either before (FC1) or

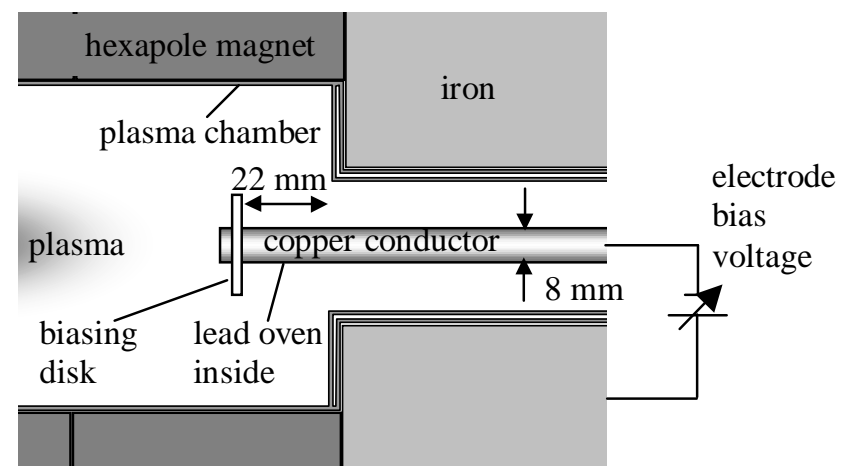

Fig. 1. Schematic drawing of the lead oven arrangement acting as a biased electrode. (The biasing disk was not used in all experiments.)

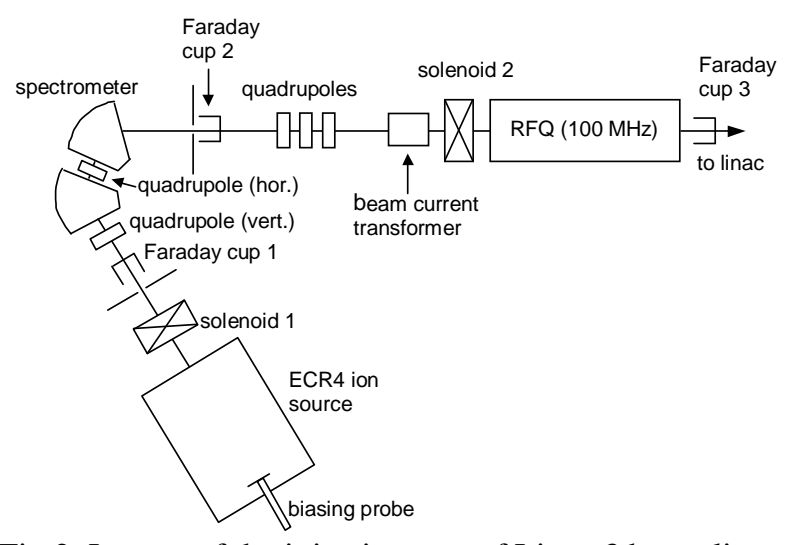

Fig.2. Layout of the injection part of Linac 3 beam line.

after mass selection (FC2) or after acceleration in the RFQ (FC3). FC1 and 2 show the full ion beam pulse whilst FC3

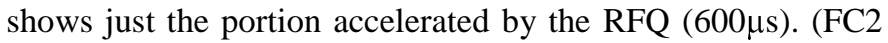
always gives a higher signal compared to FC3 due to poor electron suppression.) The total ion current at FC1 is about $1.5 \mathrm{~mA}$ during the main pulse (RF on) and increases (over about $300 \mu \mathrm{s}$ ) to about $8 \mathrm{~mA}$ after the RF is switched off. Depending on the mode of the afterglow [8] and on the tuning of the source, the current either decreases exponentially over more than $10 \mathrm{~ms}$ or ends in a sharp break down after 1-2 ms. The time structure of the FC2 current is very similar to FC1 for the higher lead charge states, but shows no afterglow increase for the $\mathrm{O}^{2+}\left(+\mathrm{Pb}^{26+}\right)$.

During normal operation the electrode and the plasma chamber are at the same potential and sparking occurs above $300 \mathrm{~V}$ due to insulation problems. Pulsing of the electrode was also tried, with variation of the voltage, the pulse length and the timing of the bias relative to the end of the RF pulse. The response to a change of the bias voltage was, within some $\mu \mathrm{s}$, too short to change the charge state distribution in the ECR 
plasma (Fig. 3). The yield increase must, therefore, be due to improved extraction conditions or an increased electron density, and not due to additional ion production (except possibly for $\mathrm{O}^{+}$).

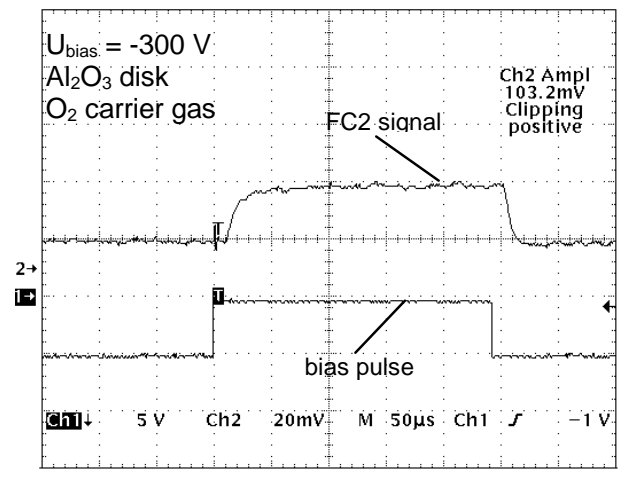

Figure 3. Time response to bias pulse in the main pulse region.

In addition to negative bias voltages, a positive pulse of up to $+300 \mathrm{~V}$, was applied after the RF-off flank and for different times. The idea was to facilitate the ejection of the electrons from the plasma, and to repel the positive plasma towards the extraction. No significant enhancement of the ion current was seen either with or without a metallic or insulating $\left(\mathrm{Al}_{2} \mathrm{O}_{3}\right)$ disk (just a small noise reduction).

While these bias tests were underway, the floating potential of the source was measured with a high impedance probe while under full RF power and with the extraction voltage off. Figure 4 shows that after the plasma ignites, the co-axial electrode rapidly rises to around $400 \mathrm{~V}$ with respect to the plasma chamber and continues rising during the heating phase to $500 \mathrm{~V}$. This potential collapses almost instantaneously, with the removal of the RF heating (1300 W), to a few volts during the afterglow pulse.

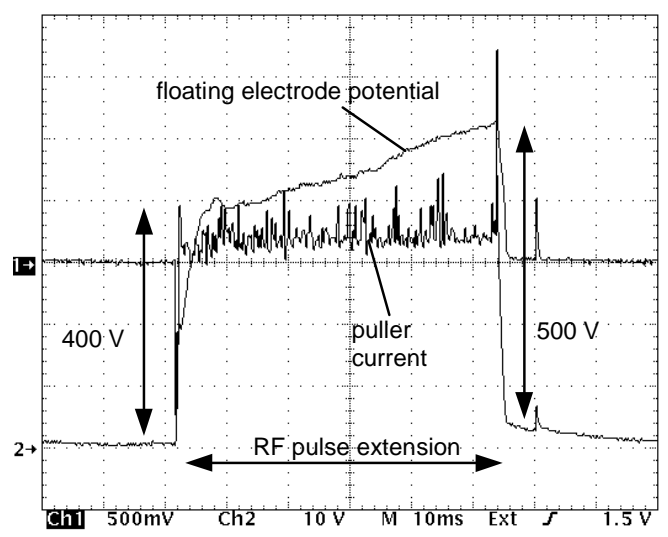

Fig. 4. Change of floating potential with RF pulse.

With the floating potential, an average electron temperature of 80 to $100 \mathrm{eV}$ [9] can be calculated. The Bohm energy is assumed to be about one half of the electron temperature i.e. about $50 \mathrm{~V}$, a value which would influence the emittance of the source in a positive way [10].
With normal extraction $(20 \mathrm{kV})$, the floating potential of the biased probe was $\sim 100 \mathrm{~V}$. A resistor of $<1 \mathrm{k} \Omega$ was necessary to keep the probe potential below $\sim 1 \mathrm{~V}$ during the microwave pulse or to discharge the (ion) current of about $1.5 \mathrm{~mA}$ (equivalent to the total extracted ion current). Larger resistors would create a bias voltage [11].

It was considered that the electrode used in the first tests [11] was possibly too small. The electrode surface was increased by the addition of metallic or dielectric disks to the coaxial line. Without a disk and zero bias the maximum $\mathrm{Pb}^{27+}$ ion current after the RFQ (FC3) was $94 \mu \mathrm{A}$, at the beginning of these tests.

1) A $20 \mathrm{~mm}$ diameter Ta disk was mounted at the end of the tube. This made the conditioning and operation of the ion source very difficult and the RF tuning had to be adjusted for a high reflected power of about $150 \mathrm{~W}$. Obviously the disk interfered with the propagation of the RF into the cavity. All settings of the source parameters were very sharp and the $\mathrm{Pb}^{27+}$ ion current (FC3) was $<60 \mu \mathrm{A}$.

2) The disk was replaced by one made from pure $\mathrm{Al}_{2} \mathrm{O}_{3}$. Conditioning was accompanied by gas bursts from the disk and needed much more time than usual, but the final operation of the source was similar to without disk. At zero bias, the maximum $\mathrm{Pb}^{27+}$ current in $\mathrm{FC} 3$ was $75 \mu \mathrm{A}$.

3) A triangular shaped Ta-disk was mounted oriented either parallel or in opposition to the hexapole loss lines. Tuning was much easier compared to the full disk, but, at zero bias, the maximum $\mathrm{Pb}^{27+}$ current was $84 \mu \mathrm{A}$ (parallel), or $64 \mu \mathrm{A}$ (opposite).

4) Finally the source was run without disk and zero bias, but the maximum $\mathrm{Pb}^{27+}$ current was only $74 \mu \mathrm{A}$ after intensive optimisation (compared to $94 \mu \mathrm{A}$ at the start of the experiments). A rebuilt and cleaned standard source could be trained after one week to $83 \mu \mathrm{A}$.

For all geometries a similar reaction to the timing of the bias voltage pulse was found:-

If the bias pulse is active only during the RF pulse, there is a gain in ion current for the $\mathrm{O}^{2+}+\mathrm{Pb}^{26+}$ for all cases. $\mathrm{Pb}^{27+}$ improved for the $\mathrm{Al}_{2} \mathrm{O}_{3}$, had an unstable gain for both orientations of a triangular Ta disk and no gain at all without disk (Fig. 5). The gain during the main pulse had no influence on the afterglow if the biasing pulse was switched off before or with the RF.

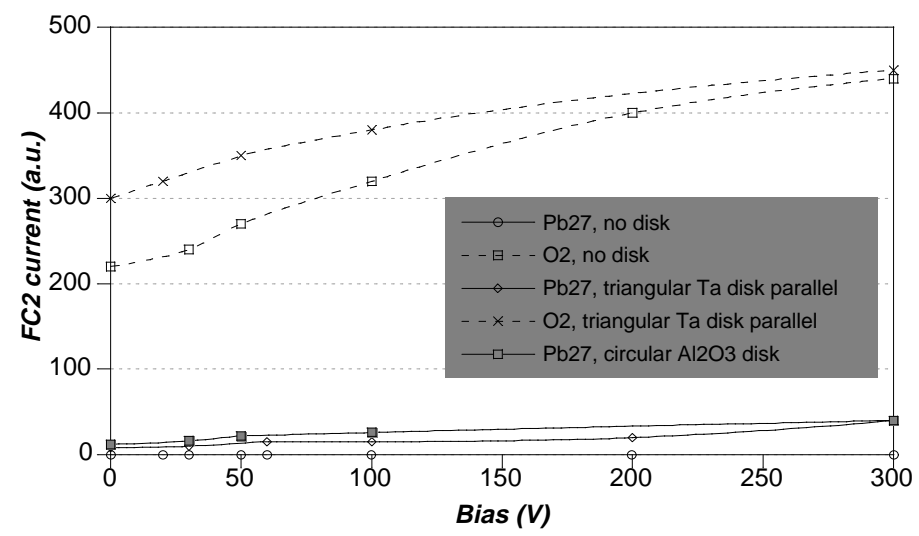

Fig. 5. Ion yields during main pulse with bias voltage. (The notations 'parallel' and 'opposite' denote the alignment of the triangular disk to the electron loss lines in the hexapole field.) 
If the bias pulse is both on the main and the afterglow region, it tends to destabilise the rising of the afterglow, creates a pre-pulse or a faster rising of the afterglow, but is then slower increasing to the maximum of the $\mathrm{Pb}^{27+}$ current.

If the bias pulse is on only during the afterglow it changes the current only during the applied time, depending on the applied voltage. In some cases a nice long stable afterglow pulse could be achieved (Fig. 6).

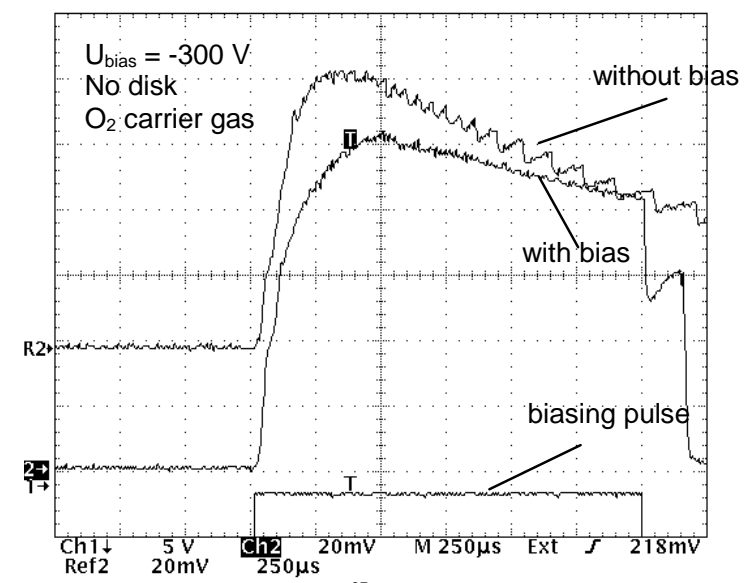

Fig. 6. Afterglow pulse of $\mathrm{Pb}^{27+}$ on $\mathrm{FC} 2$. The yield increase is evident, and the pulse less noisy.

In Fig. 7 the afterglow current in FC2 is shown depending on the bias voltage for the different geometries. Usually the ion current increases by $<10 \%$ at around $-15 \mathrm{~V}$ (depending on the disk) and decreases to a minimum at about $-30 \mathrm{~V}$. Afterwards the $\mathrm{Pb}^{27+}$ current increases again, usually to values above the $0 \mathrm{~V}$ bias one (beyond the highest available voltage of $-300 \mathrm{~V})$. The same behaviour can be seen for the $\mathrm{O}^{2+}+\mathrm{Pb}^{26+}$ afterglow yield.

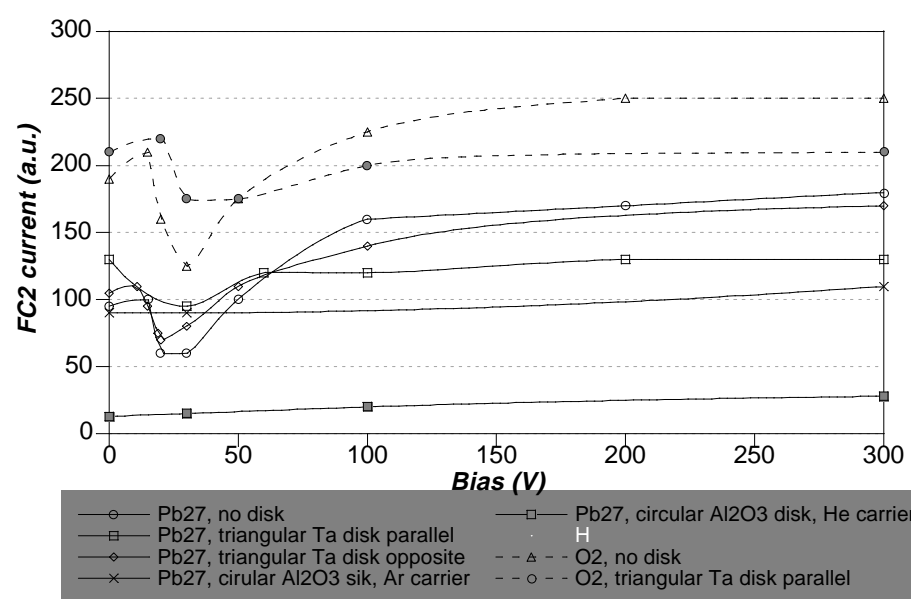

Fig. 7. Afterglow yield for $\mathrm{Pb}^{27+}$ and $\mathrm{O}^{2+}+\mathrm{Pb}^{26+}$ with bias voltage for various disk and gas combinations.

FC3 showed similar trends as FC2, but was more sensitive, sometimes beam line readjustments were necessary, i.e. a change in the source emittance occurred during the bias voltage pulse. (The solenoids after the source and in front of the RFQ had to be changed and a small adjustment of the extraction voltage $(<50 \mathrm{~V})$ was necessary for best yield). For $\mathrm{Al}_{2} \mathrm{O}_{3}$, a $10 \%$ increase for $-300 \mathrm{~V}$ bias to $82 \mu \mathrm{A}$ was found, for the triangular one from $64 \mu \mathrm{A}$ to $78 \mu \mathrm{A}$ (opposite) or $84 \mu \mathrm{A}$ to $90 \mu \mathrm{A}$ (parallel).

Other support gases, Ar and $\mathrm{He}$, were also tried. Both gave lower yields of $\mathrm{Pb}^{27+}$ ions $(57 \mu \mathrm{A}$ and $9 \mu \mathrm{A}$ respectively). For argon the increase was about $15 \%$ to $66 \mu \mathrm{A}$ (see Fig. 8).

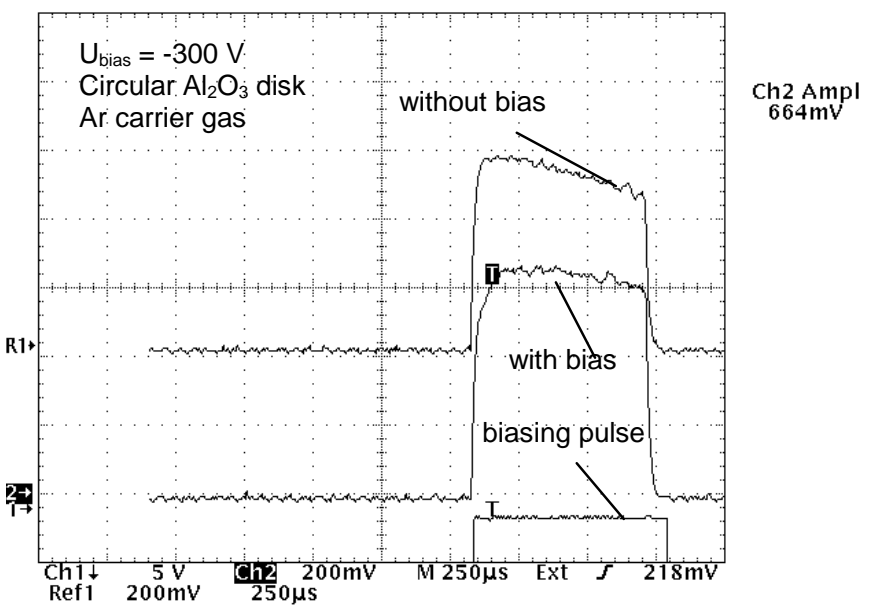

Fig. 8. $\mathrm{Pb}^{27+}$ current (FC3) for argon support gas and $-300 \mathrm{~V}$ bias. The current increases from 57 to $66 \mu \mathrm{A}$.

\section{Conclusions and Discussion}

Similar tests to those described in this paper have resulted in ion current improvements for ECR ion sources operating in pulsed or CW mode [4,5]. For the CERN source operating in afterglow mode, no significant current increases have been observed in any of these or previous experiments $[7,8,11]$. Instead, changes to the source usually result in a less stable behaviour. Any relative current increase does not improve the optimum found in the original configuration following finetuning the source. There seems to be an absolute maximum $\mathrm{Pb}^{27+}$ ion current under stable operation conditions. The source performance may already be optimised, i.e. an enhanced production of highly charged ions, or a more rapid extraction of the ions, only result in instabilities or break down of the afterglow reservoir. Consequently, the experience gained from CW sources seems not to be directly applicable to an ECR source running in afterglow mode.

There is no possibility to extract a higher ion pulse in a shorter time by applying electric potentials inside the ECR source. The only promising way is the PuMa method of a fast pulsed magnetic field [12], but this would imply a totally new source design.

Could higher afterglow currents be extracted from out of the next generation of ECR sources with higher RF frequency and/or magnetic field? Fuller understanding of the afterglow performance of an ECR ion source is needed.

\section{References}

[1] H.D. Haseroth. Proc 1995 Particle Accelerator Conference, Dallas, IEEE, VI (1996) p.411

[2] G. Melin, F. Bourg, P. Briand, J. Debernardi, M. Delaunay, R. Geller, B.Jacquot, P. Ludwig, T. K. N'Guyn, L. Pin, M. Pontonnier, J. C. Rocco, F. Zadworny, "Some Particular 
Aspects of the Physics of the ECR Source for Multicharged Ions", Rev. Sci. Instrum. 61 (1990) p.236

[3] L.R. Evans, "LHC Accelerator Physics and Technology Challenges”, Proc. 1999 Particle Accelerator Conf., New York 1999, p 21

[4] S. Runkel, K. E. Stiebing, O. Hohn1, V. Mironov, G. Shirkov, A. Schempp, H. Schmidt-Boecking, "Time-resolved measurements of the Biased-Disk Effect at the Frankfurt $14 \mathrm{GHz}$ Electron-Cyclotron-Resonance-Ion-Source”, Proc. 14th ECR Workshop, Geneva, 1999

[5] T. Nakagawa, S. Biri, T. Chiba, A. Goto, M. Hemmi, E. Ikezawa, N. Inabe, T.Kageyama, O. Kamigaito, M. Kase, M. Kidera, Y. Miyazawa and Y. Yano, "Further development of RIKEN 18 GHz ECRIS", Proc. $6^{\text {th }}$ European Part. Accelerator Conf., 1998, Stockholm, p.1418-1421

[6] P. Briand, R. Geller, H. Haseroth, C. Hill, K. Langbein, "Utilizing the ECR Afterglow to Increase the Intensity of Highly Charged Ions in the CERN Accelerator Complex", Proc. $11^{\text {th }}$ Int. Workshop on ECR Ion Sources, Groningen, 1993, KVI Report 996, p.132 (1993)

[7] C.E. Hill, K. Langbein, "Experiments on a $14.5 \mathrm{GHz}$ ECR Source", Proc. $7^{\text {th }}$ Int. Conf. on Ion Sources, Taormina, Italy, 1997, Rev. Sci. Instrum. 69, 643, 1998

[8] C. E. Hill, K. Langbein, "Experiments on a 14.5 GHz ECR Source", Proc. $18^{\text {th }}$ Int. Linac Conf., Geneva, 1996, CERN 9607, p 366 (1996)

[9] A.T.Forrester, "Large Ion Beams", Wiley, New York, 1988, p 64

[10] P. Spaedtke, "2D and 3D Simulations of Ion Beam Extraction from the ECR Ion Source" Proc. 14th ECR Workshop, Geneva, 1999

[11] C.E. Hill and F. Wenander "Experiments on the Pulsed Afterglow Operation of an ECR Ion Source“, Proc. 14th ECR Workshop, Geneva ,1999

[12] C. Muehle, U. Ratzinger, G. Jost, K. Leible, S. Schennach, B.H. Wolf, "Pulsed magnetic fiele-electron cyclotron resonance ion source operation" Proc. $6^{\text {th }}$ Int. Conf. On Ion Sources Whistler Canada 1995, Rev. Sci. Instrum. 67, p. 1331 (1996) 